schützen (vgl. Ziltener 1999: 133). Zu diesem Zweck wurden im EWS die Wechselkurse der teilnehmenden Länder mit einer gewissen Bandbreite festgeschrieben, wenn der Kurs an die Grenzen der Bandbreiten stieß, waren die zentralen Notenbanken zur Intervention verpflichtet (vgl. Huffschmid 1994: 43).

Neben dem EWS gab in dieser Phase keine großen integrationspolitischen Fortschritte. Die Mitgliedstaaten waren mit den Wirtschaftskrisen beschäftigt, wobei sie eher auf nationale als auf gemeinsame europäische Strategien setzten (vgl. Mazey 1996: 32). Überdies war die Entscheidungsfindung in einer durch die Norderweiterung von 1973 heterogener gewordenen Gemeinschaft (1981 begann mit Griechenland die Süderweiterung) nicht leicht, da das Einstimmigkeitserfordernis des Luxemburger Kompromisses noch immer in Kraft war. Festzuhalten bleibt die Gründung des Europäischen Rates im Jahr 1974, eine Institutionalisierung der bereits vorher stattfindenden Treffen der Staats- und Regierungschefs, die zum Ziel hatten, die Grundlinien der Politik der Gemeinschaft festzulegen. Weiterhin ist die erste direkte Wahl des Europäischen Parlaments vom 7.-10.6.1979 zu nennen, die selbiges deutlich aufwertete (vgl. Weidenfeld 2006: 22). Insgesamt machte zum Ende dieser Phase der Begriff 〉Eurosklerose〈 die Runde, worunter das Zusammenspiel ökonomischer Stagnation und gesunkener Handlungsfähigkeit intergouvernementaler Gremien verstanden wird (vgl. Ziltener 1999: 127).

Zuletzt der Blick auf die politische Situation in den Mitgliedstaaten: Mit dem Regierungswechsel zu Margaret Thatcher in Großbritannien setzte 1979 ein politischer Wandel hin zu liberal-konservativen Regierungen ein, dem zu Beginn der 1980er Jahre alle Mitgliedsländer folgten (vgl. Gillingham 2003: 136ff). Nur in Frankreich war eine gegenteilige Entwicklung festzustellen: Aus den Wahlen von 1981 ging eine sozialistisch-kommunistische Koalitionsregierung unter Staatspräsident Mitterand hervor, die zumindest in ihren ersten Jahren eine expansive Wirtschaftspolitik und Sozialisierungsprogramme verfolgte (vgl. Ziltener 1999: 137).

\title{
Intendierter Einfluss der europäischen Vorgaben auf die Interventionsmöglichkeiten der Mitgliedstaaten und ihre Handlungsfähigkeit
}

Zur Untersuchung der Frage, ob mit Hilfe der europäischen Vorgaben die nationalstaatlichen Interventionsmöglichkeiten eingeschränkt oder ausgebaut werden sollten, werden diese vier großen Bereichen zugeordnet: Zum einen erfolgte eine Koordinierung im Hinblick auf die Schaffung einer Wirtschafts- und Währungsunion. Die Wirtschaftskrisen er- 
forderten ein gemeinsames Vorgehen bei der kurzfristigen Wirtschaftspolitik. Die mittelfristige Wirtschaftspolitik wurde unter anderem im Rahmen der Schaffung des Gemeinsamen Marktes koordiniert. Die Überwindung der Arbeitslosigkeit wird zwar sowohl im Rahmen der kurz- wie der mittelfristigen Wirtschaftspolitik angesprochen, da es sich um eines der wichtigsten Probleme dieser Phase handelt und - im Gegensatz zum ebenfalls zentralen Problem der Inflation - viele ausgabenrelevante Maßnahmen empfohlen wurden, wird diesem Problem ein eigener Abschnitt gewidmet.

\section{Koordinierung im Hinblick auf die Wirtschafts- und Währungsunion}

Zu Beginn der Untersuchungsphase herrschte noch Optimismus bezüglich des 1969 von den Staats- und Regierungschefs ausgegebenen Ziels, eine Wirtschafts- und Währungsunion zu errichten. Eine wichtige Voraussetzung für die gemeinsame Währung wurde in der Konvergenz sowohl der Wirtschaftspolitik als auch der wirtschaftlichen Entwicklung der Mitgliedstaaten gesehen (vgl. 74/120/EWG: 16). Aus diesem Grund straffte der Rat das hierfür bereitstehende Koordinierungsinstrumentarium und fasste es teilweise strenger. Die sogenannte »Konvergenzentscheidung« von 1974 führte die bisherigen Verfahren gebündelt auf, änderte sie jedoch nur wenig: Über Konsultationen sollten nun permanent alle wirtschaftspolitischen Entscheidungen der Mitgliedstaaten vor Verabschiedung auf ihre Vereinbarkeit mit den gemeinschaftlich festgesetzten Zielen und Leitlinien überprüft werden (vgl. 74/120/EWG, Art. 9). Die Treffen des Rates zur Begutachtung und Beratung der wirtschaftlichen Situation wurden von drei auf zwölf erweitert (vgl. ebenda, Art. 1), das Verfahren zur Festlegung und Anpassung der jährlichen wirtschaftspolitischen Leitlinien in Form der Jahresberichte blieb jedoch unverändert (vgl. ebenda., Art. 2-5), gleiches galt für die ebenfalls aufgeführten Programme für die mittelfristige Wirtschaftspolitik. Neu war die Möglichkeit, zur Erreichung der vorgesehenen Ziele Entscheidungen, Richtlinien oder Empfehlungen zu verabschieden (vgl. ebenda, Art. 6). Mit der »Richtlinie über die Stabilität, das Wachstum und die Vollbeschäftigung in der Gemeinschaft« (74/121/ EWG) wählte der Rat noch im gleichen Jahr ein rechtlich verbindliches Instrument für die Koordinierung, die Mitgliedstaaten mussten sie innerhalb von 12 bzw. 24 Monaten umsetzen (vgl. ebenda, Art. 12). Die Richtlinie legte fest, dass die Mitgliedstaaten ihre Wirtschaftspolitik im Einklang mit den gemeinschaftlichen Leitlinien gestalten und bei wichtigen Maßnahmen ausdrücklich auf diese Bezug nehmen sollten (vgl. ebenda, Art. 1-2). Überdies verpflichteten 
die Mitgliedstaaten sich, auch für ein leitlinienkonformes Haushaltsgebaren von nachgelagerten Gebietskörperschaften und der sozialen $\mathrm{Si}$ cherheit zu sorgen (vgl. ebenda, Art. 8). Bei von den Leitlinien abweichender oder andere Mitgliedstaaten gefährdender Wirtschaftspolitik konnte die Kommission eine Empfehlung an den betreffenden Staat richten und eine Prüfung im Rat stattfinden (vgl. 74/120/EWG, Art. 11).

Um die verschiedenen Bereiche der Wirtschaftspolitik sinnvoll miteinander zu verbinden, wurden 1974 weiterhin die Ausschüsse für Konjunktur-, Haushalts- und mittelfristige Wirtschaftspolitik im »Ausschuss für Wirtschaftspolitik« zusammengefasst (vgl. 74/122/EWG, Art. 1). Dieser setzte sich aus je vier Mitgliedern der Kommission und jedes Mitgliedstaates zusammen (vgl. ebenda, Art. 3), er sollte die Haushaltspolitik der Mitgliedstaaten prüfen und vergleichen, einen Vorentwurf des Programms für mittelfristige Wirtschaftspolitik vorlegen, die mitgliedstaatliche Politik auf die Übereinstimmung mit den mittelfristigen Vorgaben hin überprüfen und schließlich Gründe für mögliche Abweichungen analysieren (vgl. ebenda, Art. 2).

Obwohl die beschriebenen Instrumente wie vorgesehen angewandt und das Verfahren 1979 durch die Erweiterung um regionalpolitische Aspekte noch einmal explizit anerkannt wurde, machten vor allem die beiden Wirtschaftskrisen die ehrgeizigen Pläne zur Einführung einer gemeinsamen Währung schnell zunichte. Statt der angestrebten Konvergenz wurde eine Divergenz der wirtschaftlichen Entwicklung und Politik in den Mitgliedstaaten festgestellt (vgl. 81/ 1056/EWG: 7). Die Wirtschafts- und Währungsunion verschwand zwar nicht völlig aus den Vorgaben, so bekannte der Europäische Rat 1977, diesem Ziel verbunden zu bleiben (vgl. Europäischer Rat 1977: 26) und durch eine gemeinsame Strategie zur Krisenbekämpfung Fortschritte in diese Richtung machen zu wollen (vgl. Europäischer Rat 1978a: 11). Die WWU tauchte jedoch deutlich seltener und weniger prominent auf. 1979 trat schließlich das EWS an ihre Stelle. Auch in diesem Zusammenhang wurde eine Koordinierung der nationalen Wirtschaftspolitik als sinnvoll angesehen: »Durch eine enge und wirksame Koordinierung der Wirtschafts- und Währungspolitik müßte zunächst eine ausreichende Konvergenz der Wirtschaftsentwicklung erreicht werden. Ohne diese Koordinierung wären die Bemühungen um eine größere Wechselkursstabilität vergeblich« (77/294/EWG: 28). Das System wurde zwar auch ohne eine deutliche Konvergenz eingeführt, die Bemühungen darum jedoch fortgesetzt: »Darüber hinaus vergrößert das Europäische Währungssystem die Notwendigkeit [...], Fortschritte bei der Harmonisierung von Haushaltsund Geldpolitik zu verwirklichen« (79/19/EWG: 18). 
Da die Konvergenz der Politiken und der wirtschaftlichen Entwicklungen über eine Koordinierung sowohl der kurz- wie langfristigen Wirtschaftspolitik erreicht werden sollte, werden die konkreten Inhalte und Empfehlungen dort dargestellt.

\section{Koordinierung der kurzfristigen Wirtschaftspolitik}

Bei der Koordinierung der kurzfristigen Wirtschaftspolitik ging es in dieser Phase hauptsächlich darum, abgestimmt auf die Wirtschaftskrisen zu reagieren. Das gemeinsame Vorgehen wurde in erster Linie mit der zunehmenden wirtschaftlichen Verflechtung begründet. Diese führte nach Ansicht des Rates zum einen dazu, dass sich Störungen in der Wirtschaft eines Mitgliedstaates auch auf die anderen Länder - und über den Multiplikatoreffekt wieder auf das Ursprungsland - auswirken könnten. Dies gelte für die Mitglieder der Gemeinschaft in besonderem Maße, da sie im Verhältnis zu den anderen Industrieländern besonders offen und außenhandelsabhängig seien (vgl. 82/950/EWG: 5). Zum anderen bringe die wirtschaftliche Vernetzung es mit sich, dass ein einzelner Staat nicht mehr in der Lage sei, die Konjunktur zu beleben (77/778/ EWG: 6), weil die zur Überwindung der Krise geschaffene Nachfrage im Ausland versickere, somit in hohem Maße ihrer Wirkung beraubt würde und zusätzlich »der Steuerrückfluß im Anschluß an die Haushaltsmaßnahmen geringer ausfällt und die notwendige Stärkung des Vertrauens von Investoren und Konsumenten eher schwächer ausfällt« (79/ 19/EWG S. 18). Die wirtschaftliche Verflechtung brachte nach Ansicht des Rates jedoch nicht nur Nach-, sondern auch Vorteile, da »die Mitgliedstaaten bei koordiniertem Handeln und in Zusammenarbeit mit den anderen Industrieländern die Multiplikatorwirkung ihrer Maßnahmen verstärken, das Vertrauen der Unternehmen und Verbraucher dauerhaft festigen und auf diese Weise die angestrebten Wachstumsziele verwirklichen [können]«(77/778/EWG: 6). Eine weitere Begründung für das gemeinsame Vorgehen war, dass die Gemeinschaft als ganzes einen stabilisierenden Effekt auf die Weltwirtschaft ausüben könne: »Durch eine wietere Verstärkung ihres Zusammenhalts und durch eine Verbesserung ihres inneren Gleichgewichts wird sie in der Lage sein, wirksam zur internationalen wirtschaftlichen Entwicklung und zur Festigung des Friedens in der Welt beizutragen« (Europäischer Rat 1979a: 9). Dies würde nicht zuletzt ihre internationale Rolle stärken (vgl. Europäischer Rat 1982a: 20).

Bei den Verfahren für das gemeinsame Vorgehen im Rahmen der kurzfristigen Wirtschaftspolitik handelte es sich in erster Linie um die bereits oben beschriebenen. Die wirtschaftspolitischen Leitlinien wurden 
wie vorgesehen einmal jährlich in Form der Jahresberichte verabschiedet - ebenfalls im Sinne des Verfahrens - und immer wieder an die wirtschaftliche Lage angepasst, sowohl bei der Überprüfung im ersten Quartal (hier erfolgte in den Jahren 1974-77 eine Anpassung) als auch unter der Überschrift »Anpassung der Staatshaushalte«, wobei die »quantitative[n] Orientierungsdaten für die Entwürfe der Staatshaushalte« (74/120/ EWG, Art. 3) grundsätzlich nicht veröffentlicht wurden, lediglich in den Jahren 1976-78 die im Rahmen dieser Überprüfung verabschiedeten allgemeinen Leitlinien. Die Schaffung eines schnell einsetzbaren konjunkturpolitischen Instrumentariums sowie die Aufstellung fünfjähriger Programme für Investitionen war den Mitgliedstaaten im Rahmen einer Richtlinie verbindlich auferlegt worden (vgl. 74/121/EWG, Art. 5f). 1979 sollten die Koordinierungsverfahren weiter verstärkt werden, dabei ging es darum, die Konsultationen zu verbessern, die Mitgliedstaaten zu einer sehr viel deutlicheren Verpflichtung zu bringen und ihre Beteiligung an der Koordinierung zu erweitern. In Bezug auf die Haushaltspolitik wollte man die Gleichschaltung der Zeitpläne weiter vorantreiben und die konzeptionelle Basis bei den Haushaltslinien ausweiten. Hierzu sollten von der Kommission konkrete Vorschläge ausgearbeitet werden (vgl. Rat 1979: 81f).

Trotz der umfangreichen Koordinierung nahm die Konvergenz der Mitgliedstaaten nicht zu, sondern ab (s.o.). Die Situation in den einzelnen Staaten unterschied sich, vor allem mit Blick auf Inflation und Zahlungsbilanz, so stark, dass die Vorgaben bei der Krisenbekämpfung kein identisches Vorgehen empfahlen.

»Diese Koordinierung impliziert angesichts der unterschiedlichen konjunkturellen und strukturellen Ausgangslage keine identische Politik für die Mitgliedsländer. Eine Annäherung der grundlegenden wirtschaftspolitischen Ziele ist jedoch unerläßlich, wenn man das gemeinsam Erreichte bewahren und auf dem Wege der europäischen Integration voranschreiten will« (75/C 297/01: 8).

Auch das differenzierte Vorgehen wurde europäisch koordiniert. Die Mitgliedstaaten sollten solidarisch und unterstützend vorgehen, um zu einem für alle zufriedenstellenden Ergebnis zu gelangen. In diesem Sinne fanden sich in den meisten Vorgaben zwei Empfehlungsstränge: Mitgliedstaaten mit einer geringen Inflation und einer ausgeglichenen oder überschüssigen Zahlungsbilanz sollten eine expansive Konjunkturpolitik verfolgen und auf diese Weise ihre Importe aus den Staaten steigern, die eine hohe Inflation und eine defizitäre Zahlungsbilanz hatten. Auf diese Wiese sollte es dieser zweiten Ländergruppe erleichtert werden, eine restriktive Politik mit dem Ziel der Preisstabilität zu verfolgen, die wie- 
derum auch der ersten Ländergruppe zugute kam (vgl. 77/492/EWG: 7). Was die konkreten Empfehlungen angeht, so wurde bei der expansiven Politik - die das Wachstum steigern sollte - hauptsächlich auf eine diskretionäre Nachfrageerhöhung über Haushaltspolitik bzw. deficit spending gesetzt. Die restriktive Politik hingegen, die in erster Linie auf eine Reduzierung der Inflation abzielte, sollte die Nachfrage dämpfen, wobei der Schwerpunkt hier ebenfalls auf der Haushaltspolitik lag: Zum einen wurde empfohlen, die Haushaltsdefizite, vor allem die strukturellen, zu reduzieren, über eine Dämpfung der Ausgaben oder - sofern dies nicht inflationär wirkte - Steuererhöhungen (vgl. 74/374/EWG: 3). Zum anderen sollte die Expansion der Gesamtnachfrage gebremst werden, wenn möglich mit Ausnahme bereits unterbeschäftigter Branchen (vgl. 74/C 149/01: 7). Sobald sie ihre Inflation unter Kontrolle hatten, waren auch die zuerst restriktiv agierenden Länder angehalten, eine expansive Politik zu verfolgen und zwar mit Hilfe bereits im Vorhinein vorbereiteter Investitionsprogramme (vgl. ebenda: 9).

Obwohl fast alle Vorgaben eine diskretionäre Nachfragesteuerung empfahlen, trat im Laufe dieser Periode eine immer stärkere Skepsis gegenüber dieser Vorgehensweise zutage. Bereits 1974 sollte die Konjunkturpolitik in einen längerfristigen Rahmen eingebunden werden: »Eine konjunkturpolitische Aktion zur Integrierung der Entwicklungsprozesse von neun Volkswirtschaften wird nämlich nur dann mit Aussicht auf Erfolg $\mathrm{zu}$ verwirklichen sein, wenn sie auf gemeinsame Ziele für einen längeren Zeitraum ausgerichtet wird« (74/120/EWG: 16). Begründet wurde dies zunächst damit, dass eine globale Ankurbelungspolitik zu einer weiteren Erhöhung der Inflation beitragen könne (vgl. 74/374/EWG: 2). In den Folgejahren versah der Rat die Empfehlungen einer diskretionären Stabilisierungspolitik immer wieder mit Einschränkungen, vor allem für die Länder, die bereits sehr hohe Haushaltsdefizite oder eine hohe Inflation aufwiesen. 1977 wurde die angemessene Anwendung der Konjunkturpolitik folgendermaßen beschrieben:

»[D]ie Wirtschaftstätigkeit wird in der Rezession durch Haushaltsdefizite und den privaten Verbrauch gestützt; in einer späteren Phase muß jedoch der Anteil des privaten und öffentlichen Verbrauchs abnehmen, um Raum für Investitionen zu schaffen; die Haushaltsdefizite müssen je nach Lage der Länder fühlbar zurückgehen oder gar verschwinden. Entsprechende Maßnahmen erfordern allerdings eine Differenzierung, falls die Gesamtnachfrage schwach bleiben sollte« (77/294/EWG: 20).

Die Stimmung wandte sich jedoch immer stärker gegen diese Art der Stabilisierung, insbesondere aufgrund der angespannten Haushaltslage 
vieler Mitgliedstaaten (vgl. 80/1265/EWG: 22). 1981 hielt der Europäische Rat schließlich zum ersten Mal fest, dass eine kurzfristige Stimulierung sich als unwirksam erweisen würde (vgl. Europäischer Rat 1981a: 8). Der Rat begründete dies damit, dass höhere Defizite zu höheren Zinsen führten, welche wiederum das Wachstum schwächten (vgl. 81/1056/ EWG: 6). Diese Crowding-Out-These war schon vorher erwähnt, aber nie als entscheidend angesehen worden, nun wurde in ihrem Sinne allen Mitgliedstaaten eine »gesunde« Haushaltspolitik empfohlen (vgl. Europäischer Rat 1981a: 8), in konjunkturellen Abschwüngen sollten nur noch die automatischen Stabilisatoren wirken (vgl. 82/950/ EWG: 11). Ein »Overkill« bei der Sanierung der Defizite sei jedoch zu vermeiden, da dies über das Sparparadoxon zu weiteren Defiziterhöhungen führen könne (vgl. 81/1056/EWG: 6).

Gleichzeitig mit der wachsenden Skepsis gegenüber der kurzfristigen Stabilisierung erhielt die Inflationsbekämpfung einen immer höheren Stellenwert. Während sie zu Beginn noch gleichrangig mit den anderen wirtschaftspolitischen Zielen wie Wachstum und Beschäftigung war, wurde sie zunehmend als prioritär angesehen, da sie »auf mittlere und lange Sicht eine Voraussetzung für die Lösung der Probleme des Wachstums, der Strukturveränderungen und damit der Beschäftigung [ist]« (Europäischer Rat 1979c: 8).

Die Koordinierung der kurzfristigen Wirtschaftspolitik wurde als mäßig erfolgreich bewertet, der Rat lobte zwar häufig Länder, die sich leitlinienkonform verhalten hatten (vgl. z.B. 76/916/ EWG: 6), bezeichnete die Koordinierung jedoch immer wieder als noch unzureichend: »[R]echtzeitig beschlossene und durchgeführte Gemeinschaftsmaßnahmen [hätten] es wahrscheinlich gestattet, die wirtschaftlichen und sozialen Lasten der Krise für alle zu mildern« (77/ 294/EWG: 10). Die Ursache für den mangelnden Erfolg wurde in den unterschiedlichen Situationen der Mitgliedstaaten gesehen, allerdings zeigte auch die darauf beruhende differenzierte Strategie nicht den rechten Erfolg, die restriktive Politik der Hochinflationsländer wurde nur selten von einer expansiven Politik der preisstabilen Länder aufgefangen (77/778/EWG: 3). Dennoch stellte der Europäische Rat (1981a: 7) im letzten Jahr des Untersuchungszeitraums fest »daß die Gemeinschaft unter den derzeitigen schwierigen Wirtschaftsbedingungen nach wie vor eine wesentliche Einrich-tung und ein unerläßliches Instrument zur Einschränkung der Auswirkungen der Rezession und zur Herbeiführung eines Umschwungs ist.« 


\section{Koordinierung der mittelfristigen Wirtschaftspolitik}

Auch zur Erreichung mittelfristiger Ziele ging man gemeinsam vor. Dies nahm mit der wachsenden Skepsis gegenüber der kurzfristigen Nachfragesteuerung in seiner - zumindest ergänzenden - Bedeutung $\mathrm{zu}$, denn die »klassischen Instrumente der Globalsteuerung allein reichen nicht aus, um diese Probleme zu lösen und den Zusammenhalt der Gemeinschaft zu stärken« (76/646/EWG: 2). Die mittelfristige Wirtschaftspolitik umfasste in erster Linie Struktur- und Investitionspolitik zur Förderung des Gemeinsamen Marktes, aber auch sozialpolitische Maßnahmen. Begründet wurde das gemeinschaftliche Vorgehen nicht nur mit den Vorteilen, die es für die einzelnen Staaten in Bezug auf Wachstum und Wohlstand brachte, sondern auch damit, als europäischer Wirtschaftsraum Wettbewerbsfähigkeit gegenüber den wichtigsten Konkurrenten USA und Japan zu gewinnen. Dabei ging es vor allem um die Vorteile eines großen Gemeinsamen Marktes wie auch um ein Aufholen im Bereich Technologie.

Das gemeinsame Vorgehen bestand zum einen in der Fortführung der Programme für die mittelfristige Wirtschaftspolitik, im Untersuchungszeitraum wurden das 4. und 5. Programm verabschiedet. Das 4. Programm sollte einen wirtschaftspolitischen Aktionsrahmen darstellen, die Mitgliedstaaten wurden angehalten, ihre Maßnahmen systematisch auf ihre Übereinstimmung mit den darin festgelegten Zielen zu untersuchen (vgl. 77/294/EWG: 6). Im Gegensatz zum 3. Programm wurden keine Länderleitlinien mehr aufgestellt, dafür wurden die Orientierungsdaten und Empfehlungen teilweise nach Ländern differenziert. Dies galt insbesondere für die öffentlichen Finanzen, so wurden z.B. der Kreditbedarf und die Art der Finanzierung von Defiziten für einzelne Mitgliedstaaten festgelegt. Überdies hielt der Rat es für notwendig, über quantitative Orientierungsdaten »für die großen Haushaltsblöcke, für jene Arten von Ausgaben, deren Anstieg besonders kräftig oder besonders gering ist, und für die Salden [...] zu verfügen« (77/294/ EWG S. 25). Auch in den Jahresberichten fanden sich Empfehlungen bezüglich der mittelfristigen Wirtschaftspolitik.

Zur Förderung einer gemeinsamen Technologiepolitik wurde 1974 eine Entscheidung (74/C 07/ 02) erlassen, die festlegte, wie die mitgliedstaatliche Politik in diesem Bereich koordiniert sowie Aktionen von gemeinschaftlichem Interesse durchgeführt werden sollten. Man hoffte, durch ein koordiniertes Vorgehen Doppelarbeit und gegenläufige Tendenzen zu verhindern, die Wirksamkeit der einzelstaatlichen Aktionen zu erhöhen und Kosten zu senken (vgl. ebenda, Art. 1). Als Instrumente waren Konsultationen (vgl. ebenda) und die Einrichtung eines Aus- 
schusses vorgesehen, der im Rahmen der Koordinierung unter anderem die Haushaltsposten für Wissenschaft und Technologie der Mitgliedstaaten vergleichen sollte (vgl. ebenda: 4). Auch die jährlichen wirtschaftspolitischen Leitlinien empfahlen den Mitgliedstaaten, Forschung und die Anwendung neuer Technologien zu fördern (vgl. 77/778/EWG: 7).

Weiterhin sollten die Wachstums- und Wettbewerbsfähigkeit über eine Strukturpolitik mit Hilfe von öffentlichen und privaten Investitionen erreicht werden. Zur Förderung produktiver privater Investitionen wurden Subventionen empfohlen, allerdings nur für fortschrittliche Industrien, Erhaltungssubventionen für unproduktive Branchen wurden abgelehnt (vgl. 75/361/EWG: 15; 77/340/EWG: 23). Einen Sonderfall nimmt hier die Schiffbauindustrie ein. Da sie für bedeutende Branchen, darunter einige mit hochentwickelter Technologie, unerlässlich sei, erlaubte der Rat die Gewährung von Erhaltungs- und anderen Subventionen (vgl. z.B. 78/338/EWG: 13). ${ }^{94}$ Ein gemeinsames Vorgehen bei der Beihilfenpolitik wurde allgemein als sinnvoll angesehen, um einen Überbietungswettlauf (vgl. 77/778/EWG: 7), sowie eine Segmentierung der Märkte und nationale Diskriminierungen zu verhindern (vgl. 81/1056/EWG: 10). Hinsichtlich der öffentlichen Investitionen sollte die »Haushaltspolitik [...] stärker als in der Vergangenheit als Instrument der Wachstumspolitik und zur Investitionsförderung eingesetzt werden« (77/492/ EWG: 8). Dazu wurde eine Umstrukturierung der Staatshaushalte hin zu investiven Ausgaben empfohlen (vgl. 80/1265/EWG: 22), wobei auch zwischen den öffentlichen Investitionen eine Prioritätenänderung vorgenommen werden sollte: von den in den letzten 20 Jahren stark geförderten Investitionen in Schulen, Universitäten und Autobahnen hin zu solchen, die - wie FuE, Ausbildung und neue Kommunikationstechnologien - unmittelbar zur wirtschaftlichen Entwicklung beitrugen (vgl. 82/ 950/EWG: 7). Der Ausbau von Infrastruktur und öffentlichen Transportmitteln sollte auch einen positiven Beitrag für den Gemeinsamen Markt leisten (vgl. ebenda). Detaillierte Regelungen gab es für den Bereich der Eisenbahnunternehmen: Ziel war es, dieses Verkehrsmittel und insgesamt den Verkehrsmarkt zu fördern ohne auf diese Weise den Wettbewerb zu verzerren (vgl. 75/327/EWG: 3). Daher wurde angestrebt, die Ausgleichszahlungen für die Transportpflicht $\mathrm{zu}$ harmonisieren (vgl. ebenda, Art. 9) und die finanziellen Beziehungen zwischen Staat und Eisenbahnen möglichst gleich und transparent zu gestalten (vgl. 74/C 111/01: 2).

Den Staaten wurde demnach eine bedeutende Rolle bei der mittelfristigen Wirtschaftspolitik zugeschrieben. Allerdings wurde die Höhe der

94 Die Begründung war die gleiche wie in Phase I dargestellt. 
öffentlichen Ausgaben in einigen Ländern als problematisch angesehen, da dies die Gefahr von Steuererhöhungen und damit Inflationsrisiken in sich berge und dem Markt Ressourcen entzogen würden (vgl. 77/294/ EWG: 24). Andererseits ging der Rat auch davon aus, dass der Bedarf an öffentlichen Gütern weiter ansteigen würde - neben den oben genannten öffentlichen Gütern mit allokativer Funktion auch an öffentlichen Gütern mit distributiver Funktion (die jedoch deutlich seltener empfohlen wurden) wie Gesundheit, soziale Sicherung, Umweltschutz und Stadtsanierung (vgl. 80/1265/EWG: 23). Hier wurde eine Prioritätensetzung und optimale Anpassung der Ausgaben an die »legitimen kollektiven Bedürfnisse« (77/294/EWG: 24) empfohlen.

Diese Empfehlungen leiten bereits über zu den sozialpolitischen Vorgaben, die sich auch auf die Sozialleistungen bezogen. So stellte der Rat im ersten sozialpolitischen Aktionsprogramm von 1974 fest, dass wirtschaftliche Expansion kein Selbstzweck sei, sondern dazu dienen müsse, Lebensqualität und -standard zu verbessern. Daher sprach er sich für die »Einführung einer geeigneten Abstimmung der Politiken der Mitgliedstaaten auf dem Gebiet der sozialen Sicherung, insbesondere im Hinblick auf die Förderung ihrer Annäherung auf dem Wege des Fortschritts« (74/C 13/01: 3) aus. Die sozialen Sicherungssysteme sollten schrittweise ausgeweitet und Verfahren eingeführt werden, welche die Sozialleistungen an den wachsenden Wohlstand anpassten (vgl. ebenda). Im gleichen Jahr setzten die Staats- und Regierungschefs das Ziel, »die Sozialleistungen der einzelnen Mitgliedstaaten im fortschrittlichen Sinn zu harmonisieren, ohne jedoch die bestehenden Sozialordnungen zu vereinheitlichen« (Konferenz der Staats- bzw. Regierungschefs 1974: 8). Langfristig wurde eine Erhöhung der Umverteilungswirkungen der öffentlichen Ausgaben, vor allem bei den oft degressiv wirkenden Sozialversicherungen angestrebt (vgl. 77/294/EWG: 24). Zum Ende der Phase wurden die - auch aufgrund der immer höheren Arbeitslosigkeit - angestiegenen Sozialausgaben jedoch auch als problematisch angesehen, da sie das Wachstum hemmen könnten (vgl. 82/950/EWG: 8). So sollten die »Haushaltsposten für die soziale Sicherheit [...] daraufhin überprüft werden, ob ihr Wachstum begrenzt, ihre Finanzierung neu überdacht und ihre Wirksamkeit verbessert werden kann« (81/ 1056/EWG: 7) und den Ländern wurde empfohlen, keine langfristigen Ausgabenverpflichtungen, insbesondere in Bezug auf Renten, einzugehen (vgl. 82/950/ EWG: 9).

Obwohl die Koordinierung der mittelfristigen Wirtschaftspolitik in den 1970er Jahren immer stärker in den Mittelpunkt rückte, wurden ihre Erfolge als bescheiden angesehen. Das 3. Programm für die mittelfristige Wirtschaftspolitik wurde als Misserfolg bezeichnet (vgl. 77/294/ 
EWG: 9f), was die Mitgliedstaaten jedoch nicht daran hinderte, ein sehr umfangreiches 4. Programm zu verabschieden. Probleme gab es erst beim 5. Programm aus dem Jahr 1982. Die Mitgliedstaaten konnten sich nur mehr auf zwei Seiten einigen (zum Vergleich: 3.: 55 Seiten, 4.: 28 Seiten), auf denen sie festhielten, dass es unterschiedliche Auffassungen bei den Zielprioritäten und vor allem der Frage, ob eine kurzfristige Stimulierung oder konsequente Stabilisierung notwendig sei, gebe (vgl. 82/ 534/EWG: 11). Daher wurde dieses Programm nur als »eine Art Orientierungsrahmen« verstanden, der keine konkreten Maßnahmen für die Mitgliedstaaten enthielt (vgl. ebenda). Auch der Jahresbericht von 1982 stellte fest, dass die jüngsten Koordinationserfahrungen »nicht gerade ermutigend« (82/950/EWG: 5) seien:

»Ein Zeichen für diese Schwierigkeiten war in den letzten Jahren das allmähliche Abrücken von quantifizierten Zielsetzungen für die einzelnen wirtschaftspolitischen Ziele wie Produktion, Preisstabilisierung, Beschäftigung. Es hat sich gezeigt, daß die wirtschaftspolitischen Steuerungsinstrumente unzureichend waren, um vielfältige Ziele glaubwürdig festzulegen« (ebenda: 9).

Erklärt wurde der Misserfolg damit, dass zwar Einigkeit über die strukturellen Missstände bestehe, aber Uneinigkeit über die Strategien zu ihrer Beseitigung (vgl. ebenda: 5f).

\section{Koordinierung des Kampfes gegen die Arbeitslosigkeit}

Der Anstieg der Arbeitslosigkeit kann in den 1970er Jahren insbesondere auf die Wirtschaftskrisen, aber auch auf den Anstieg der Erwerbsbevölkerung zurückgeführt werden. Letztere wuchs demographisch bedingt um ein Prozent pro Jahr, zusätzlich aus sozialen Gründen, da immer mehr Menschen arbeiten wollten (vgl. 82/950/EWG: 7). In diesem Zusammenhang entwickelte sich vor allem die Jugendarbeitslosigkeit zu einem großen Problem. "Sowohl aus sozialen wie auch wirtschaftlichen und politischen Gründen kann die Gemeinschaft ein Andauern der hohen Arbeitslosigkeit [...] nicht hinnehmen« (77/294/EWG: 4).

Mit einem gemeinschaftlichen bzw. abgestimmten Vorgehen sollte erreicht werden, dass »auf einzelstaatlicher und gemeinschaftlicher Ebene eine Beschäftigungspolitik erreicht wird, mit der die Arbeitslosigkeit wirkungsvoller bekämpft werden kann« (80/C 168/01: 79). Teilweise wurde eine Wiedererlangung der Vollbeschäftigung bis 1980 als Ziel gesetzt (z.B. 77/294/ EWG: 17). Zu diesem Zweck sollte 
»[d]ie Gemeinschaft [...] in die Lage versetzt werden, die Auswirkungen der verschiedenen Gemeinschaftspolitiken auf die Beschäftigung schon in der Konzeptionsphase systematisch zu beurteilen, die Beschäftigungspolitiken auf Gemeinschaftsebene besser zu koordinieren und die Gemeinschaftsmaßnahmen im Bereich der Wirtschaftspolitik und im sozialen und beschäftigungspolitischen Bereich zu harmonisieren« (Europäischer Rat 1980a: 9).

Das gemeinsame Vorgehen erfolgte zum einen im Rahmen der oben beschriebenen Koordinierungsverfahren, zusätzlich sollte es ein »energisches und koordiniertes Gemeinschaftsvorgehen auf dem Arbeitsmarkt« (Konferenz der Staats- bzw. Regierungschefs 1974: 8) geben, um vorrangige Ziele festzulegen und die nationalen Maßnahmen zur Bekämpfung der Arbeitslosigkeit abzustimmen (vgl. ebenda). 1980 wurden zum ersten Mal eigenständige Leitlinien für eine Arbeitsmarktpolitik der Gemeinschaft verabschiedet (80/C 168/01), hierfür war jedoch kein jährlicher Rhythmus vorgesehen.

Die Vorgaben empfahlen eine ganze Reihe von Instrumenten: »Schaffung wirtschaftlich gesunder Arbeitsplätze, Anpassung der Arbeitskräfte an die neuen Gegebenheiten, Erleichterung der Last, die mit der zeitweilig unvermeidlich hohen Arbeitslosigkeit verbunden ist « (80/ 1265/EWG: 20). Die Lösung wurde demnach sowohl auf der Mikro- wie der Makroebene gesucht. Makroökonomisch ging es zum einen um die Bekämpfung der konjunkturellen Arbeitslosigkeit über die beschriebene diskretionäre Nachfragepolitik. Weiterhin wurde empfohlen, durch eine aktive Wirtschafts-, Beschäftigungs- und Investitionspolitik neue Arbeitsplätze zu schaffen (vgl. Europäischer Rat 1978a: 12). Die Mitgliedstaaten sollten die öffentlichen Mittel zugunsten von solchen Ausgaben umschichten, »welche arbeitsschaffende Investitionen direkt oder indirekt und nach Möglichkeit in selektiver Weise anregen« (76/916/EWG: 11). Wie bei der mittelfristigen Wirtschaftspolitik warnte der Rat auch hier davor, Beihilfen für überholte Strukturen zu zahlen (77/340/EWG: 23). Ein gemeinsames Vorgehen sollte vermeiden, »daß sich die nationalen Aktionen bei der sektoralen Orientierung widersprechen und damit zum Teil unwirksam werden« (77/340/EWG: 22). Neben beschäftigungsfördernden öffentlichen Investitionen wurden auch direkte Arbeitsmarkthilfen (81/1056/EWG: 7) sowie Arbeitsbeschaffungsprogramme - sowohl allgemein als auch für gewisse Gruppen (Jugendliche) und in gewissen Sektoren (Energie) - empfohlen, wobei auf den engen haushaltspolitischen Spielraum hingewiesen wurde (80/1265/EWG: 26). Überdies sollte eine Lohnmäßigung einen Beitrag zur Inflationsbekämpfung leisten, indem sie auf diese Weise oder auch direkt die Ertragslage und Investitionsneigung der Unternehmen steigerte (vgl. z.B. 
75/C 297/01: 9). Potential für neue Arbeitsplätze sah der Rat vor allem im Dienstleistungsbereich (vgl. 80/67/EWG: 25).

Bei der - mikroökonomischen - Arbeitsmarktpolitik lag der Schwerpunkt auf dem Bereich Bildung. Auch hier war eine Abstimmung der nationalen Politiken vorgesehen (vgl. 74/C 13/01: 2), die Staaten wurden aufgefordert, die Möglichkeiten der Aus- und Weiterbildung durch erhebliche Investitionen auszubauen (vgl. 74/374/EWG: 2) und die derzeitigen Strukturen den neuen wirtschaftlichen und sozialen Erfordernissen anzupassen (vgl. 80/C 168/01: 80). Um die Weiterbildung attraktiver zu machen, sollten die Staaten in Zeiten der Umschulung angemessene Lohnersatzleistungen zur Verfügung stellen: »Insbesondere soll während der Periode der Umschulung dem Arbeitnehmer ein Einkommensniveau garantiert werden, das nicht $\mathrm{zu}$ sehr unter demjenigen in seinem ursprünglichen Beruf liegt« (74/374/EWG: 2). Dies sollte auch die Mobilität der Arbeitskräfte fördern, vor allem in Richtung der Wachstumsbranchen (vgl. 75/ 361/EWG: 15). Weiterhin empfahlen die Vorgaben eine Verkürzung oder zumindest Flexibilisierung der Arbeitszeiten. Ausgabenpolitisch relevant ist die flexiblere Altersgrenze für den Renteneintritt, in einer eigenen Entscheidung legte der Rat fest, dass die Mitgliedstaaten keine prohibitiv hohen Rentenabschläge bei der Frühpensionierung einführen durften (vgl. 82/857/ EWG: 27). Uneingeschränkt wurde jedoch nur Teilzeitarbeit empfohlen (vgl. Europäischer Rat 1978a: 12), ansonsten wurde bei den arbeitszeitpolitischen Empfehlungen immer auch vor höheren Kosten für die Arbeitgeber bzw. - bei der Altersgrenze für die Rente - den Staat gewarnt (vgl. bspw. 80/67/EWG: 25), ebenso sollten negative Auswirkungen auf die Flexibilität der Arbeitsmärkte vermieden werden (vgl. 82/950/EWG: 9). Flexibilisierung wurde ansonsten selten angesprochen, 1977 wurde ein Abbau von Arbeitsmarkthindernissen aller Art, d.h. administrativ, steuerlich, sozialversicherungstechnisch und rechtlich, empfohlen (vgl. 77/ 340/EWG: 23). Schließlich wurde zum Ende der Phase der Gedanke geäußert, eine Absenkung von Sozialbeiträgen könne die Einstellungsanreize für Unternehmer erhöhen (vgl. 81/ 1056/EWG: 11). Insgesamt sollten die »Gemeinschaftsorgane für den koordinierten Einsatz der einzelstaatlichen Instrumente [im Bereich Arbeitsmarktpolitik, A.K.] sorgen und dabei deren Wirkung auf die Beschäftigung berücksichtigen« (77/778/EWG: 7). 


\section{Schlussfolgerungen}

Nach der Darstellung der Vorgaben und Empfehlungen in den vier groBen Bereichen wird nun noch einmal zusammenfassend analysiert, ob mit den europäischen Vorgaben das Ziel verfolgt wurde, die nationalstaatlichen Interventionsmöglichkeiten einzuschränken oder auszubauen.

Im Bereich Allokation sollten die Interventionsmöglichkeiten ausgebaut werden: Für die Subventionen wurden viele Ausnahmeregelungen vom vertraglich festgelegten Verbot verabschiedet, sowohl für den Schiffbau als auch zur Förderung produktiver Branchen im Rahmen der Strukturpolitik. Staatliche Interventionen in Bezug auf öffentliche Güter mit allokativer Funktion, insbesondere Forschung und Entwicklung sowie Bildung und Infrastruktur, wurden sehr positiv gesehen: Mit ihrer Hilfe sollten Wachstum und Wettbewerbsfähigkeit angeregt und so auch mehr Beschäftigung geschaffen werden. Bei diesen Vorgaben gab es im Verlauf der 1970er Jahre kaum Änderungen, wobei teilweise auf den eingeschränkten Haushaltsspielraum hingewiesen wurde, weshalb eher eine Umschichtung der Staatsausgaben als ihre Erhöhung empfohlen wurde. Die Verbindlichkeit der Vorgaben reichte von den recht unverbindlichen jährlichen Leitlinien im Rahmen der Jahresberichte - wobei die Mitgliedstaaten durch eine Richtlinie dazu verpflichtet waren, sich an diese zu halten (vgl. 74/121/EWG, Art. 1) - bis hin zu verbindlichen Richtlinien und Verordnungen für den Schiffbau und die Eisenbahnen.

Im Bereich Distribution änderte sich die Absicht hinter den Vorgaben: Zu Beginn der Phase sollten die nationalstaatlichen Interventionsmöglichkeiten - vor allem über die Jahresberichte - ausgebaut werden: Die Staaten wurden aufgefordert, öffentliche Güter mit distributiver Funktion (Gesundheit, Stadtsanierung etc.) bereitzustellen und die Sozialleistungen dem wachsenden Wohlstand anzupassen. Im Laufe der Zeit nahmen diese Vorgaben jedoch ab und verkehrten sich Anfang der 1980er Jahre teilweise in ihr Gegenteil: Die Staaten sollten sparen und die Sozialleistungen senken, um auf diese Weise die wirtschaftliche Leistung und die Einstellungsbereitschaft der Arbeitgeber zu erhöhen.

Der deutlichste Wandel von einem beabsichtigen Ausbau zu einer Einschränkung von Interventionsmöglichkeiten ist im Bereich Stabilisierung zu erkennen. Im ersten Jahr der Untersuchungsphase verpflichtete der Rat die Mitgliedstaaten per Richtlinie, ein schnell einsetzbares konjunkturpolitisches Instrumentarium aufzubauen. Im Rahmen der differenzierten, solidarischen Strategie wurde in den rechtlich weniger verbindlichen Jahresberichten einigen Staaten eine expansive, interventionistische Stabilisierungspolitik über den Haushalt relativ uneingeschränkt empfohlen. Anderen Staaten - mit hoher Inflation, hohen Haushalts- und 
Zahlungsbilanzdefiziten - sollten hingegen eine restriktive Politik verfolgen. Mit wachsender Skepsis gegenüber der diskretionären Stabilisierungspolitik nahmen auch die Einschränkungen für die erste Staatengruppe zu, Interventionen wurden nur mehr bei gesunder Haushaltslage und am Ende nur noch regelgeleitet (über die automatischen Stabilisatoren) empfohlen.

Für alle drei Bereiche - Allokation, Distribution und Stabilisierung gab es weniger quantifizierte Ziele, was die (öffentliche) Überwachung der Umsetzung erschwerte. Somit wandelte sich in dieser Phase die Absicht hinter den europäischen Vorgaben: In den ersten Jahren sollte das gemeinsame Vorgehen die Interventionsmöglichkeiten der Mitgliedstaaten, die vor allem im Bereich der Stabilisierung durch die wirtschaftliche Verflechtung als eingeschränkt wahrgenommen wurden, wieder ausbauen: »Anders gesagt soll durch ein Vorgehen auf Gemeinschaftsebene der Prozeß umgekehrt werden, der infolge der zunehmenden Einbindung der Wirtschaft der Mitgliedstaaten in die Europäische Gemeinschaft und die Weltwirtschaft die Wirksamkeit einer rein nationalen Wirtschaftspolitik unterhöhlt hat« (79/19/EWG: 17). Zum Ende der Phase gab es immer mehr Vorgaben, welche die mitgliedstaatlichen Interventionsmöglichkeiten einschränken sollten, neben dem Bereich der zu dieser Zeit als unwirksam angesehenen Stabilisierung auch bei den Sozialausgaben.

\section{Staatsverständnis}

Auch für diese zweite Integrationsphase wird eine qualitative Inhaltsanalyse durchgeführt, um daraus Rückschlüsse auf das den Leitlinien zugrunde liegende Staatsverständnis zu ziehen. Da für diesen Zeitraum insgesamt 32 Dokumente analysiert werden konnten, ist eine Unterteilung in zwei Dokumententypen möglich: Die 13 codierten Schlussfolgerungen des Europäischen Rates (bzw. bis 1974: Konferenz der Staats- bzw. Regierungschefs) werden getrennt von den anderen Dokumenten (als ER) ausgewiesen. ${ }^{95}$ Dies ist insofern sinnvoll, als dass die Schlussfolgerungen in dieser Zeit sehr kurz und meist eher allgemein gehalten waren und daher nur wenige Kategorienzuordnungen möglich sind. Bei den restlichen 19 Dokumenten handelt es sich überwiegend um wirtschafts-

95 Es handelt sich hierbei um folgende Dokumente: Konferenz der Staatsbzw. Regierungschefs 1974, Europäischer Rat 1976a, Europäischer Rat 1976b, Europäischer Rat 1978b, Europäischer Rat 1979a, Europäischer Rat 1979b, Europäischer Rat 1979c, Europäischer Rat 1980a, Europäischer Rat 1980b, Europäischer Rat 1981a, Europäischer Rat 1981b, Europäischer Rat 1982a, Europäischer Rat 1982c. 